\title{
Inhibition of endothelin receptors' phosphorylation for therapy of brain metastasis and glioblastoma
}

\section{$\underline{\text { Isaiah J. Fidler }}$}

Department of Cancer Biology, Metastasis Research Center, The University of Texas M. D. Anderson Cancer Center, Houston, Texas, USA

Background: The pathogenesis of metastasis is regulated by the interaction of unique tumor cells with specific host organ microenvironments which tumor cells exploit. A prime example is brain metastasis. Histologic examination of experimental and clinical specimens of human brain metastases and glioblastoma reveals that the lesions are surrounded and infiltrated by activated astrocytes expressing glial fibrillary acidic protein. Cells in the metastases and glioblastoma express high levels of phosphorylated endothelin receptors (ETR) A and B. Astrocytes cocultured with tumor cells enhance expression of phosphorylated ETR in tumor cells leading to upregulation of multiple genes, including the survival genes GSTA5, BCL2-L1, and TWIST1, and protect the cells from all tested chemotherapeutic agents. Knocking down the survival genes with specific SiRNA rendered the tumor cells sensitive to chemotherapy.
We have identified the signal that astrocytes send to tumor cells as endothelin-1, -2. Phosphorylation of ETR A and B on tumor cells was followed by activation of Akt, MAPK, followed by upregulation of the survival genes. We hypothesized that the use of ETR antagonist will prevent upregulation of survival genes and render tumor cells sensitive to anticycling agents. Luciferase-tagged breast cancer, lung cancer, and glioblastoma cells were implanted in the brains of nude mice. Administration of the dual endothelin receptor inhibitor Macitentan ${ }^{\circledR}$ combined with Taxotere or Temozolomide produced complete destruction of existing breast cancer and lung cancer brain metastasis, and glioblastoma in nude mice. These data clearly demonstrate that astrocytes influence the biological behavior of tumor cells in the brain and reinforce the conclusion that the organ microenvironment must be taken into consideration for the design of effective therapy.

The studies described here began with a hypothesis that astrocytes protect tumor cells from cytotoxic agents. In vitro studies using cocultures of astrocytes and tumor cells were followed by successful in vivo therapy studies. These translational studies stimulated ongoing phase I clinical trials of glioblastoma. 